\title{
TWISTORS AND NEARLY AUTOPARALLEL MAPS
}

\author{
Sergiu I. Vacaru* \\ Institute of Applied Physics, \\ Academy of Sciences of Moldova, \\ 5 Academy str., Chişinău, 2028, Republic of Moldova \\ and \\ Sergiu V. Ostaf \\ Department of Mathematics and Physics, \\ Tiraspol State University (evacuated in Chişinău), \\ 5 Iablochkin str., Chişinău, 2062, Republic of Moldova
}

July 3, 2021

\begin{abstract}
The purpose of the present paper is to investigate the problem of definition of twistors on generic curved spaces. Firstly, we consider nearly geodesic ( autoparallel ) maps of (pseudo)-Riemannian spaces . Secondly, we shall define nearly autoparallel twistor equations which are compatible on nearly conformally flat spaces. Finally, we shall study nearly autoparallel twistor structures generating curved spaces and vacuum Einstein spaces.
\end{abstract}

*E-mail: lises@cc.acad.md 


\section{Introduction: Spinors and maps of curved spaces with deformation of connections}

Our geometrical constructions will be realized on pairs of 4-dimensional (pseudo)-Riemannian spaces $(V, \underline{V})$ with signature $(-+++)$ and $1-1$ local maps of spaces $f: V \rightarrow \underline{V}$, given by functions $f^{\underline{\mu}}(x)$ of smoothly class $\mathcal{C}^{r}(U),(r>2, r=\omega$ for analytic functions ) and theirs inverse functions $f^{\mu}(\underline{x})$ with corresponding non-zero Jacobians in every point $x=\left\{x^{\mu}\right\} \subset U \subset V$ and $\underline{x}=\left\{\underline{x}^{\mu}\right\} \subset \underline{U} \subset \underline{V} \quad(U$ and $\underline{U}$ are open regions $)$. We shall attribute regions $U$ and $\underline{U}$ to a common for a given $\mathrm{f}$-map coordinate system when every point $q \in U$, with coordinates $x^{\mu}(q)$, is mapped into a point $q \subset U$ with the same coordinates $x^{\mu}=\underline{x}^{\mu}(\underline{q})=x^{\mu}(\underline{q})$, i.e. $f: x^{\mu}(q) \rightarrow x^{\mu}(\underline{q})$. We note that all calculations in this work will be local in nature and taken to refer to common coordinates, for given $\mathrm{f}$-maps, on open regions of spaces into considerations.

The metric tensor, connection, differential operator and tetrads (frames) on $U$ and $\underline{U}$ are correspondingly denoted as $g_{\mu \nu}(x), \Gamma_{\mu \nu}^{. \alpha}(x), D_{\mu}$ and $h_{\mu}^{a}(x)$, where

$$
g_{\mu \nu}(x)=h_{\mu}^{a}(x) h_{\nu}^{b}(x) \eta_{a b}, \quad \eta_{a b}=\text { const }
$$

and, respectively, $\underline{g}_{\mu \nu}(x) \equiv g_{\underline{\mu} \underline{\underline{\nu}}}(x), \underline{\Gamma}_{\mu \nu}^{. \alpha}(x) \equiv \Gamma_{\underline{\mu} \underline{\mu} \underline{\alpha}}^{. \alpha}(x), \underline{D}_{\mu}=D_{\underline{\mu}}$ and $\underline{h}_{\mu}^{a}(x)=$ $h_{\underline{\mu}}^{a}(x)$, where

$$
\underline{g}_{\mu \nu}(x)=\underline{h}_{\mu}^{a}(x) \underline{h}_{\nu}^{b}(x) \eta_{a b}, \quad \eta_{a b}=\text { const }
$$

(Greek indices $\mu, \nu, \underline{\mu}, \underline{\nu}, \ldots$ run from 0 to 3 ). Throughout the present paper we shall use the terminology and definitions of abstract and coordinate tensor and spinor index formalisms described in monographs [1, 2]. For simplicity, we shall write Latin symbols $a, b, \underline{a}, \underline{b}, \ldots$ for both abstract and tetrad indices implying that in the first case Latin indices are abstract labels and in the second case (for decompositions on tetrads ) we shall specify their explicit values $(a, b, \ldots=0,1,2,3)$. We consider spinor decompositions of metrics (1) and (2) :

$$
g_{\mu \nu}=\sigma_{\mu}^{A A^{\prime}}(x) \sigma_{\nu}^{B B^{\prime}}(x) \epsilon_{A B} \epsilon_{A^{\prime} B^{\prime}},
$$

where $\sigma_{\mu}^{A A^{\prime}}(x)=h_{\mu}^{a}(x) \sigma_{a}^{A A^{\prime}}, \sigma_{a}^{A A^{\prime}}=$ const, are Infeld-van der Waerden coefficients and $\epsilon_{A B}=-\epsilon_{B A}, \epsilon_{A^{\prime} B^{\prime}}=-\epsilon_{B^{\prime} A^{\prime}}\left(A, B, A^{\prime}, B^{\prime}=0,1\right)$ are spinor metrics

$$
\left(\underline{g}_{\mu \nu}=\underline{\sigma}_{\mu}^{A A^{\prime}}(x) \underline{\sigma}_{\nu}^{B B^{\prime}}(x) \epsilon_{A B} \epsilon_{A^{\prime} B^{\prime}}\right.
$$


where $\underline{\sigma}_{\mu}^{A A^{\prime}}(x)=\sigma_{\underline{\mu}}^{A A^{\prime}}(x)=\underline{h}_{\mu}^{a}(x) \sigma_{a}^{A A^{\prime}}$; if necessary we shall write, for example, $\underline{\epsilon}_{A B}, \underline{\epsilon}_{A^{\prime} B^{\prime}}, \underline{\sigma}_{a}^{\bar{A}}, A^{\prime}, \underline{\omega}^{A}$ or $\underline{\xi}_{A^{\prime}}^{A B}$ in order to point that these spin-tensor values are associated to the spinor decomposition (4) on space $\underline{V}$ ).

For mutual transformations of tensor and spinor indices one introduces inverse Infeld-van der Waerden coefficients $\sigma_{A A^{\prime}}^{\mu}(x)$ and $\underline{\sigma}_{A A^{\prime}}^{\mu}(x)$, for example, $B^{\alpha}=\sigma_{A A^{\prime}}^{\alpha} B^{A A^{\prime}}$ and $\underline{A}_{B B^{\prime}}=A_{\underline{\mu}} \sigma_{B}^{\frac{\mu}{B^{\prime}}}$.

Covariant derivation of spinors on $V$ is defined by using spin coefficients $\gamma_{\ddot{A} A^{\prime} B}^{C}$ and $\gamma_{\ddot{A} A^{\prime} B^{\prime}}^{C^{\prime}}$ :

$$
D_{A A^{\prime}} \xi^{B}=\sigma_{A A^{\prime}}^{\mu}(x) D_{\mu} \xi^{B}=\partial_{A A^{\prime}} \xi^{B}+\gamma_{\ddot{A} A^{\prime} C}^{B} \xi^{B}
$$

and $D_{A A^{\prime}} \xi_{B^{\prime}}=\sigma_{A A^{\prime}}^{\mu}(x) D_{\mu} \xi_{B^{\prime}}=\partial_{A A^{\prime}} \xi_{B^{\prime}}-\gamma_{\ddot{A} A^{\prime} B^{\prime}}^{C^{\prime}} \xi_{C^{\prime}}$

In a similar manner spin coefficients $\underline{\gamma}_{A A^{\prime} B}^{\ldots . C}$ and $\underline{\gamma}_{A A^{\prime} B^{\prime}}^{\ldots C^{\prime}}$ are considered in order to define covariant derivation of spinors on $\underline{V}$.

Here we present formulas interrelating spin coefficients $\gamma_{\mu B}^{. C}$ and $\gamma_{\mu B^{\prime}}^{. .} \cdot C^{\prime}$ with Christoffel symbols in the case of orthonormalized bases (when $\epsilon_{A^{\prime} B^{\prime}}=\epsilon_{A B}=$ $\left.\left(\begin{array}{cc}0 & 1 \\ -1 & 0\end{array}\right)\right):$

$$
\begin{aligned}
& \gamma_{\mu B}^{. . C}=\Gamma_{\mu B C^{\prime}}^{\cdots . C C^{\prime}}-\frac{1}{2} \sigma_{B B^{\prime}}^{\nu} \partial_{\mu} \sigma_{\nu}^{C B^{\prime}}, \quad \gamma_{\mu B^{\prime}}^{. C^{\prime}}=\Gamma_{\mu B B^{\prime}}^{\ldots . C^{\prime}}-\frac{1}{2} \sigma_{B B^{\prime}}^{\nu} \partial_{\mu} \sigma_{\nu}^{B C^{\prime}} \\
& \left(\gamma_{\underline{\mu} B}^{. C C}=\Gamma_{\underline{\mu} B C^{\prime}}^{\cdots C C^{\prime}}-\frac{1}{2} \sigma_{B B^{\prime}}^{\nu} \partial_{\underline{\mu}} \sigma_{\underline{\nu}}^{. C B^{\prime}}, \quad \gamma_{\underline{\mu}}^{. \cdot \frac{C}{B^{\prime}}}=\Gamma_{\underline{\mu} B B^{\prime}}^{\cdots B C^{\prime}}-\frac{1}{2} \sigma_{B B^{\prime}}^{\underline{\nu}} \partial_{\underline{\mu}} \sigma_{\underline{\nu}}^{. B C^{\prime}}\right) .
\end{aligned}
$$

and, inversely, for $\sigma_{a}^{A A^{\prime}}=$ const,

$$
\Gamma_{B B^{\prime} C C^{\prime}}^{. . A A^{\prime}}=\gamma_{B B^{\prime} C}^{\ldots A} \epsilon_{C^{\prime}}^{. A^{\prime}}+\bar{\gamma}_{B B^{\prime} C^{\prime}}^{\ldots A^{\prime}} \epsilon_{C}^{. A} \quad\left(\underline{\Gamma}_{B B^{\prime} C C^{\prime}}^{\ldots . A A^{\prime}}=\underline{\gamma}_{B B^{\prime} C^{\prime}}^{\ldots A} \underline{\epsilon}_{C^{\prime}}^{A^{\prime}}+\bar{\gamma}_{B B^{\prime} C^{\prime}}^{\ldots . A_{C}^{\prime}} \epsilon_{C}^{. A}\right)
$$

where $\epsilon_{A}^{B}=\delta_{A}^{B}, \epsilon_{C^{\prime}}^{B^{\prime}}=\delta_{A}^{B}$ and $\bar{\gamma}_{B B^{\prime} C^{\prime}}^{\ldots}$ denotes complex conjugation of $\left.\gamma_{B B^{\prime} C^{\prime}}^{A}\right)$.

Our first objective in this paper is to study deformations of spinor objects in result of superposition of local 1-1 maps $f: V \rightarrow \underline{V}$ with deformation of connection

$$
\Gamma_{\beta \gamma}^{. . \alpha}(x)=\Gamma_{\beta \gamma}^{. . \alpha}(x)+P_{\beta \gamma}^{. \alpha}(x),
$$

and, in consequence of formulas (5), deformations of spin coefficients, for example,

$$
\underline{\gamma}_{\mu B}^{. . C}(x)=\gamma_{\mu B}^{. . C}(x)+{ }^{\star} \gamma_{\mu B}^{C}(x)
$$


where $P_{\ddot{\beta} \gamma}^{. \alpha}(x)$ and ${ }^{\star} \gamma_{\mu B}^{. . C}=P_{\mu B C^{\prime}}^{\ldots C C^{\prime}}-\frac{1}{2}\left(\sigma_{B B^{\prime}}^{\nu} \partial_{\mu} \sigma_{\underline{\nu}}^{C B^{\prime}}-\sigma_{B B^{\prime}}^{\nu} \partial_{\mu} \sigma_{\nu}^{C B^{\prime}}\right)$ are called the deformation tensor and, respectively, the deformation spin tensor. Deformations of the covariant derivation operator, cased by splittings of type (6), or (7), will be denoted as

$$
\underline{D}_{\mu}=D_{\mu}+{ }^{\star} D_{\mu}, \quad \underline{D}_{A A^{\prime}}=D_{A A^{\prime}}+{ }^{\star} D_{A A^{\prime}}
$$

In a particular case of conformal maps $c: U \rightarrow \underline{U}$, when

$$
\underline{g}_{a b}=\Omega^{2} g_{a b}, \underline{\epsilon}_{A B}=\Omega \epsilon_{A B}, \underline{\epsilon}_{A^{\prime} B^{\prime}}=\Omega \epsilon_{A^{\prime} B^{\prime}},
$$

$\Omega(x)$ is a nonzero real function on $U$,

$$
{ }^{\star} D_{\mu}=\Omega^{-1} D_{\mu} \Omega=D_{\mu} \ln \Omega \text {. }
$$

Conformal transforms are largely used, for example, in twistor [2] and conformal field theories.

We note that there are classes of 1-1 local maps with deformation of connection ((6) or (7)) more general then that for conformal maps (9) (see, for example, $(n-2)$-projective spaces [3], nearly geodesic maps, ng-maps [4], nearly autoparallel maps, na-maps, of spaces with torsion and nonmetricity $[5,6]$, of fiber bundles [7] and of Finsler and Lagrange spaces [8] ). In works $[5,9,10,11]$ we have proposed to apply ng- and na-maps for definition of conservation laws on curved spaces. $\mathrm{Na}$-maps were used for definition of nearly autoparallel twistors in connection to a possible twistor-gauge interpretation of vacuum gravitational fields [7, 11-14].

The second objective is the investigation of na-map deformations of twistor equations [2] (for our purposes written on space $\underline{V}$ )

$$
\underline{D}_{A^{\prime}}^{(A} \underline{\omega}^{B)}=\frac{1}{2}\left(\underline{D}_{A^{\prime}}^{A} \underline{\omega}^{B}+\underline{D}_{A^{\prime}}^{B} \underline{\omega}^{A}\right)=0,
$$

where ( ) denotes symmetrization.

Because for uncharged twistors

$$
\underline{D}^{A^{\prime}(C} \underline{D}_{A^{\prime}}^{A} \underline{\omega}^{B)}=-\underline{\Psi}_{. . D}^{C A . B} \underline{\omega}^{D},
$$

where

$$
\underline{C}_{a b c d} \equiv \underline{\Psi}_{A B C D} \underline{\epsilon}_{A^{\prime} B^{\prime}} \underline{\epsilon}_{C^{\prime} D^{\prime}}+\underline{\bar{\Psi}}_{A^{\prime} B^{\prime} C^{\prime} D^{\prime}} \underline{\epsilon}_{A B} \underline{\epsilon}_{C D}
$$


is the conformal Weyl tensor on space $\underline{V}$, there is a hard compatibility condition for twistor equations (10), namely, $\underline{\Psi}_{A B C D \underline{\omega}^{D}}=0$, which characterizes, for example, conformally flat spaces. That is why a rigorous mathematical, generally accepted, definition of twistors was possible only for conformally flat spaces and this fact is the main impediment to a twistor interpretation of general gravitational fields ( see details in [2] ).

Our main idea $[11,7,13,14]$ was to define twistors not on generic curved spaces $V$, where twistor equations are incompatible, but to remove the problem on auxiliary conformally flat (or more simply, flat $\underline{M}$ ) background spaces $\underline{V}$, interrelated with the fundamental space-time $V$ by means of chains of namaps (nearly conformal maps, nc-maps). On space $\underline{M}$ twistor equations (10) become compatible; we can define twistors in a standard manner as pairs of spinors, $\underline{Z}^{\alpha}=\left(\underline{\omega}^{A}, \underline{\pi}_{A}\right)$. Then, transferring $\underline{Z}^{\alpha}$ on $V$, by using nc-maps, we can define nearly autoparallel twistors, na-twistors, as satisfying na-twistor equations, being na-images of equations (10). For simplicity , in this paper we shall restrict ourselves only with nearly geodesically flat, ng-flat, spaces $V$, which admit ng-maps to Minkowski space $\underline{M}$. We shall analyze conditions when na-twistor equations contain information on vacuum Einstein fields.

\section{$2 \quad$ Nearly geodesic maps and spinors}

The aim of this section is to present a brief introduction into the geometry of ng-flat spaces. We shall specify basic ng-map equations and invariant conditions [4] to the case of vacuum gravitational fields on $V$. Proofs are mechanical, but, in most cases, rather tedious calculations, similar to those presented in $[4,13]$. They are omitted.

2.1. Definition of $n g-m a p s$. Let parameterize curves on $U \subset V$ by functions $x^{\mu}=x^{\mu}(\eta), \eta_{1}<\eta<\eta_{2}$, with corresponding tangent vector field defined as $u^{\mu}=\frac{d x^{\mu}}{d \eta}$.

Definition 1.A curve $l$ is called a geodesic on $V$ if its tangent vector field satisfies autoparallel, a-parallel, equations :

$$
u D u^{\alpha}=u^{\beta} D_{\beta} u^{\alpha}=\rho(\eta) u^{\alpha},
$$

where $\rho(\eta)$ is a scalar function. 
We note that for (pseudo)-Riemannian spaces the extremal curves, the geodesics, coincide with the straightest curves, a-parallels, and that is why we shall use term geodesics for both classes of curves (for spaces with locally isotropic or anisotropic torsion and nonmetricity we have started with aparallel equations $[5-8]$ ).

On space $\underline{V}$ we consider a new class of curves : Let curve $\underline{l} \subset \underline{V}$ is given parametrically as $x^{\alpha}=x^{\alpha}(\eta), \eta_{1}<\eta<\eta_{2}, u^{\alpha}=\frac{d x^{\alpha}}{d \eta} \neq 0$. We say that a 2 dimensional distribution $E_{2}(\underline{l})$ is coplanar along $\underline{l}$ if in every point $x \in \underline{l}$ it is defined a 2-dimensional vector space $E_{2}(x) \subset T_{x} \underline{V} \quad\left(T_{x} \underline{V}\right.$ is the tangent space to $x \in \underline{V})$ and every vector $\underline{p}^{\mu}\left(x_{(0)}^{\nu}\right) \subset E_{2}(\underline{l}), x_{(0)}^{\mu} \in \underline{l}$, rests contained in the same distribution after parallel transports along $\underline{l}$, i.e. $p^{\alpha}\left(x^{\beta}(\eta)\right) \subset E_{2}(\underline{l})$.

Definition 2. A curve $\underline{\underline{l}}$ is called a nearly geodesic on space $\underline{V}$ if a coplanar along $\underline{l}$ distribution $E_{2}(\underline{l})$ containing the tangent to $\underline{l}$ vector field $u^{\alpha}(\eta)$ is defined.

$\mathrm{Ng}-$ maps are introduced [4] according the

Definition 3 Nearly geodesic maps, ng-maps, are local 1-1 mappings of (pseudo)-Riemannian spaces, $n g: V \rightarrow \underline{V}$, changing every geodesic $l$ on $V$ into nearly geodesic $\underline{l}$ on $\underline{V}$.

Let a geodesic $l \subset U$ is given by functions $x^{\alpha}=x^{\alpha}(\eta), u^{\alpha}=\frac{d x^{\alpha}}{d \eta}, \eta_{1}<\eta<$ $\eta_{2}$, satisfying equations (12). We suppose that to the geodesic $l$ corresponds a nearly geodesic $\underline{l} \subset \underline{U}$ given by the same parametrization in a common for a chosen ng-map local coordinate system on $U$ and $\underline{U}$. This requirement is satisfied if and only if vectors $u^{\alpha}, \underline{u}_{(1)}^{\alpha}=u \underline{D} u^{\alpha}$ and $\underline{u}_{(2)}^{\alpha}=u \underline{D} u_{(1)}^{\alpha}$ are linearly depended in every point $x \in \underline{U}$, i.e.

$$
\underline{u}_{(2)}^{\alpha}=\underline{a}(\eta) u^{\alpha}+\underline{b}(\eta) \underline{u}_{(1)}^{\alpha}
$$

for some scalar functions $\underline{a}(\eta)$ and $\underline{b}(\eta)$. Putting splitting (6) into expressions for $\underline{u}_{(1)}^{\alpha}$ and $\underline{u}_{(2)}^{\alpha}$ on $U$ and from the just presented linear dependence we obtain :

$$
u^{\beta} u^{\gamma} u^{\delta}\left(D_{\beta} P_{\gamma \delta}^{. . \alpha}+P_{\beta \gamma}^{. . \alpha} P_{\gamma \delta}^{. . \tau}\right)=b u^{\gamma} u^{\delta} P_{\gamma \delta}^{. . \alpha}+a u^{\alpha},
$$

where $b(\eta, u)=\underline{b}-3 \rho$ and

$$
a(\eta, u)=\underline{a}+\underline{b} \rho-u^{b} \partial_{b} \rho-\rho_{2}
$$

are called the deformation parameters of ng-maps. 
2.2. Classification of $n g-m a p s$. $\mathrm{Ng}$-maps were classed [4] by considering possible polynomial dependencies on $u^{\alpha}$ of deformation parameters (15). We shall consider maps $n g: V \rightarrow \underline{V}$ satisfying reciprocity conditions (when $n g^{-1}$ : $V \rightarrow V$ is also an ng-map). This requirement is fulfilled if

$$
P_{\alpha(\beta}^{. . \tau} P_{\gamma \delta)}^{\ldots \alpha}=d_{(\beta} P_{\gamma \delta)}^{. . \tau}+c_{(\alpha \beta} \delta_{\gamma)}^{\tau},
$$

for a vector, $d_{\beta}$, and tensor, $c_{\alpha \beta}$, on $V$.

Theorem 1 Four classes of ng-maps are characterized by corresponding parametrizations of deformation tensors and basic equations :

-for trivial ng-maps, geodesic maps (or $\pi_{(0)}$-maps)

$$
P_{\beta \gamma}^{. . \alpha}(x)=\psi_{(\beta} \delta_{\gamma)}^{\alpha},
$$

where $\delta_{\beta}^{\alpha}$ is Kronecker symbol and $\psi_{\beta}=\psi_{\beta}(x)$ is the covariant vector fields; -for $\pi_{(1)}-$ maps $P_{\beta \gamma}^{. \alpha}(x)$ is the solution of equations

$$
3 D_{\alpha} P_{\beta \gamma}^{. . \tau}=2 R_{(\beta . \gamma) \alpha}^{\tau}-2 \underline{R}_{(\beta . \gamma \alpha)}^{\tau}+6 b_{(\alpha} P_{\beta \gamma)}^{. \tau \tau}+6 a_{(\alpha \beta} \delta_{\gamma)}^{\tau} ;
$$

- for $\pi_{(2)}-$ maps

$$
P_{\alpha \beta}^{. . \tau}=2 \phi_{(\alpha} \delta_{\beta)}^{\tau}+2 \sigma_{(\alpha} F_{\beta)}^{\tau},
$$

where $F_{\beta}^{\alpha}=F_{\beta}^{\alpha}(x)$ satisfies conditions $F_{\alpha}^{\beta}=F_{\delta}^{\alpha}=e \delta_{\delta}^{\beta},(e= \pm 1)$,

$$
\partial_{[\beta} F_{\gamma]}^{\tau} F_{\lambda}^{\gamma}-\partial_{[\lambda} F_{\gamma]}^{\tau} F_{\beta}^{\gamma}=0
$$

([ ] denotes antisymmetrization) and solves equations

$$
D_{\beta} F_{\alpha}^{\tau}+\mu_{\gamma} F_{\alpha}^{\gamma} \delta_{\beta}^{\tau}-\mu_{\alpha} F_{\beta}^{\tau}=0
$$

for a covariant vector field $\mu_{\gamma}=\mu_{\gamma}(x)$;

-for $\pi_{(3)}-$ maps

$$
P_{\gamma \delta}^{. . \tau}=2 \psi_{(\gamma} \delta_{\delta)}^{\tau}+\sigma_{\gamma \delta} \varphi^{\tau}
$$

where the contravariant vector field $\varphi^{\tau}=\varphi^{\tau}(x)$ satisfies equations

$$
D_{\alpha} \varphi^{\beta}=\nu \delta_{\alpha}^{\beta}+\mu_{\alpha} \varphi^{\beta}
$$

for some scalar, $\nu=\nu(x)$, covariant vector, $\mu_{\gamma}=\mu_{\gamma}(x)$ and $\varphi_{\nu}=\varphi_{\nu}(x)$ symmetric tensor $\sigma_{\alpha \beta}=\sigma_{\alpha \beta}(x)$ fields. 
We emphasize that for $\varphi_{\mu}=\frac{\partial \varphi}{\partial x^{\mu}}=g_{\mu \nu} \varphi^{\nu}$ and $\sigma_{\alpha \beta}(x)=g_{\alpha \beta}(x)$ we obtain a particular case of conformal maps, $\pi_{3(C)}: \underline{g}_{\mu \nu}=e^{2 \varphi} g_{\mu \nu}$ (the so called concircular maps [15]).

\subsection{Invariant criterions for ng-flat spaces.}

Definition 4. (Pseudo)-Riemannian space $V$ is $n g$-flat if it admits a map $n g: V \rightarrow \underline{M}$.

We shall consider four classes of na-flat spaces denoted respectively as $\pi_{(i)}$-flat spaces, where $(i)=((0),(1),(2),(3))$.

It is significant that na-maps are characterized by corresponding invariant conditions for values being similar to Thomas parameters and Weyl tensor (the invariants for conformal maps [16]). Here we present the criterions for a space $V$ to be ng-flat .

Proposition 1 : For ng-flat spaces there are satisfied the next conditions : -for $\pi_{(0)}$-spaces

$$
W_{\alpha . \gamma \delta}^{. \beta}=R_{\alpha . \gamma \delta}^{. \beta}-\frac{2}{3} R_{\alpha[\gamma} \delta_{\delta]}^{\beta}=0
$$

-for $\pi_{(1)}-$ spaces

$$
3 D_{\gamma} P_{\alpha \beta}^{. . \tau}=2 R_{(\alpha \beta) \gamma}^{. \tau}+6 b_{(\alpha} P_{. \beta \gamma)}^{\tau}+6 a_{(\alpha \beta} \delta_{\gamma)}^{\tau}
$$

-for $\pi_{(2)}$-spaces

$$
\hat{W}_{\alpha . \beta \delta}^{\cdot \tau}=\hat{R}_{\alpha . \beta \gamma}^{. \tau}+\frac{2}{5} \hat{R}_{[\beta \gamma]} \delta_{\alpha}^{\tau}-\frac{2}{15}\left[4 \hat{R}_{\alpha[\beta} \delta_{\gamma]}^{\tau}+\delta_{[\gamma}^{\tau} \hat{R}_{\beta] \alpha}\right]=0,
$$

where

$$
\begin{aligned}
\hat{R}_{\alpha . \beta \gamma}^{. \tau}= & -R_{\alpha . \beta \gamma}^{\cdot \tau}+e F_{\delta}^{\tau}\left(F_{\delta}^{\lambda} R_{\lambda . \beta \gamma}^{\delta}+2 R_{\lambda . \alpha[\gamma}^{\delta} F_{\beta]}^{\lambda}-2 D_{[\gamma} F_{\beta]}^{\delta}-\right. \\
& \left.2 e F_{\epsilon}^{\lambda} D_{(\alpha} F_{\beta)}^{\epsilon} D_{\lambda} F_{\gamma}^{\delta}+2 e F_{\epsilon}^{\lambda} D_{(\alpha} F_{\gamma)}^{\epsilon} D_{\lambda} F_{\beta)}^{\delta}\right)
\end{aligned}
$$

and

$$
\begin{gathered}
\hat{R}_{\epsilon \tau}=-R_{\epsilon \tau}+e F_{\alpha}^{\delta}\left(F_{\epsilon}^{\beta} R_{\beta . \tau \delta}^{. \alpha}+F_{\tau}^{\beta} R_{\beta . \epsilon \delta}^{\alpha}-2 D_{\epsilon} D_{[\delta} F_{\tau]}^{\alpha}-2 e F_{\beta}^{\gamma} D_{(\epsilon} F_{\tau)}^{\beta} D_{\gamma} F_{\delta}^{\alpha}+\right. \\
\left.e F_{\beta}^{\gamma} D_{(\epsilon} F_{\delta)}^{\beta} D_{\gamma} F_{\tau}^{\alpha}\right)
\end{gathered}
$$




$$
\begin{aligned}
& - \text { for } \pi_{(3)} \text {-spaces } \\
& R_{\beta \alpha \gamma \delta}=A\left(g_{\alpha \gamma} g_{\beta \delta}-g_{\alpha \delta} g_{\beta \gamma}\right)+e B\left[\varphi_{\beta}\left(g_{\alpha \gamma} \varphi_{\delta}-g_{\alpha \delta} \varphi_{\gamma}\right)-\varphi_{\alpha}\left(g_{\beta \gamma} \varphi_{\delta}-g_{\beta \delta} \varphi_{\gamma}\right)\right]
\end{aligned}
$$

where $A=-\frac{1}{2}\left[\frac{R}{3}-2\left(\frac{d \nu}{d \varphi} e \nu^{2}\right)\right]$, and $A+B+\left(\frac{d \nu}{d \varphi}+e \nu^{2}\right)=0, e= \pm 1$, for some gradient vector $\varphi_{\alpha}=\frac{d \varphi}{d x^{\alpha}}$ and scalar $\nu(\varphi)$ fields.

We note that from (28) one follows this expression for the Ricci tensor of $\pi_{3}-$ flat spaces:

$$
R_{\alpha \beta}=\left[\frac{R}{3}-\left(\frac{d \nu}{d \varphi}+e \nu^{2}\right)\right] g_{\alpha \beta}-\left[\frac{R}{3}-4\left(\frac{d \nu}{d \varphi}+e \varphi^{2}\right)\right] e \varphi_{\alpha} \varphi_{\beta} .
$$

2.4. The integrability conditions for ng-maps equations. All presented in this paper basic equations for ng-maps ( equations (17), (18) and (20)(22)) are systems of first order partial differential equations with algebraic constraints of type (19). The integrability conditions for ng-map equations have been studied in [4] and, in the language of Pffaf systems [17-20], in [5, $6,13]$. The most important conclusion made in the just mentioned works is that we can always verify, by using algebraic methods, if a given system of ng-map equations on $V$ is, or not, integrable for maps to Minkowski space. Let illustrate this for maps $\pi_{1}: V \rightarrow \underline{M}$ specified by equations

$$
3\left(D_{\gamma} P_{\alpha \beta}^{. . \tau}+P_{\alpha \beta}^{. . \epsilon} P_{\gamma \epsilon}^{. . \tau}\right)=2 R_{(\alpha . \beta) \gamma}^{. \tau}+6 a_{(\alpha \beta} \delta_{\gamma)}^{\tau}
$$

(these equations can be obtained from (14) by using auxiliary $\pi_{0}$-maps , $\bar{V} \stackrel{\pi}{\rightarrow} V \rightarrow \underline{M}$, see [4]). The integrability conditions of (31) can be written as

$$
S_{. \alpha \beta \gamma \epsilon}^{\tau}=6\left(D_{\epsilon} a_{(\alpha \beta} \delta_{\gamma)}^{\tau}-D_{\gamma} a_{(\alpha \beta} \delta_{\epsilon)}^{\tau}\right),
$$

where

$$
\begin{gathered}
S_{. \alpha \beta \gamma \epsilon}^{\tau}=-D_{\epsilon} R_{(\alpha, \beta) \gamma}^{\cdot \tau}+D_{\gamma} R_{(\alpha \beta) \epsilon}^{\tau}+6 P_{\lambda(\alpha}^{\cdot \tau} R_{\beta) \cdot \gamma \epsilon}^{\cdot \lambda}+4 R_{(\alpha . \beta)[\epsilon}^{\cdot \lambda} P_{\gamma] \lambda}^{\cdot \tau}+ \\
6 P_{\gamma \lambda}^{\cdot \tau} \delta_{(\alpha}^{\lambda} a_{\beta \epsilon)}-6 P_{\epsilon \lambda}^{\cdot \tau} \delta_{(\alpha}^{\lambda} a_{\beta \gamma)}
\end{gathered}
$$

From (32) we obtain

$$
3 D_{\gamma} a_{\alpha \beta}=\frac{1}{3} S_{. \epsilon(\alpha \beta) \gamma}^{\epsilon}-S_{. \alpha \beta \gamma \epsilon}^{\epsilon}
$$


Equations (31) and (34) forms a Cauchy system of first order partial differential equations (with coefficients given by geometrical values such as connection and curvature on $V$ ) for unknown variables $P_{\beta \gamma}^{. \alpha}$ and $a_{\alpha \beta}$.

The first set of integrability conditions for the system of equations (31) and (34) can be found from (32) by excluding covariant derivations of $a_{\alpha \beta}$ according to (34). In result we obtain integrability conditions being linear equations for deformation tensor $P_{\alpha \beta}^{. . \gamma}$. Introducing the second, third and so on sets of integrability conditions, we are, in general, able to clarify the question of existence of solutions of system (31) and (34).

We note that in a similar manner we can construct sets of integrability conditions for $\pi_{(2)^{-}},(20)$, and $\pi_{(3)}-\operatorname{map},(22)$, equations.

2.5. Spinor formalism and the ng-map theory. This question is studied in details in $[13,14]$ by introducing deformations by ng-maps of spin coefficients (7) in spinor covariant derivation operator (8). Using $\sigma$-coefficients we can transform basic ng-map equations (18), (20) and (22) and flat projectivity conditions (23), (24) and (28) into spinor form. We omit these considerations here. For our purposes it is important the fact that for every deformation of spin coefficients ${ }^{\star} \gamma_{\mu B}^{\cdot . C}(x)$ (see splitting $(7)$ ) we can define corresponding deformation tensor (see expressions (5)-(7)),

$$
P_{B B^{\prime} C C^{\prime}}^{\ldots}{ }^{\star} \gamma_{B B^{\prime} C}^{\ldots A} \epsilon_{C^{\prime}}^{A^{\prime}}+^{\star} \bar{\gamma}_{B B^{\prime}} \ldots A_{C}^{\prime} \cdot \epsilon_{C}^{A}
$$

Putting (35), for example, into (33) we obtain a system of algebraic equations, if necessary in spinor variables with a spinor representation of curvature and deformation parameters, which permits us to answer the question if, the given deformation of spin coefficients generates, or not, a map $\pi_{(1)}: V \rightarrow \underline{M}$.

Finally, in this section, we note that every curved space $V$, if corresponding conditions on differentiability of components of metric, connection and curvature on $V$ are satisfied, admits a finite chain of ng-maps, i.e. a nctransform, to Minkowski space $\underline{M}[5-9,13]$. So, it is possible a new classification of curved spaces in terms of minimal chains of ng-maps characterized by corresponding sets of invariant conditions of type (23)-(25) and (28). This ng-map classification of curved spaces differs from the well known Petrov's algebraic classification [21]. 


\section{$3 \quad$ Nearly conformal twistors}

The purpose of this section is to define twistors on ng-flat spaces.

3.1 Spinor equations for massless fields with spin $\frac{m}{2}(m=0,1,2, \ldots)$ and twistor equations. Let spinor $\phi_{A B \cdots L}$ has $m$ indices and is symmetric:

$$
\phi_{A B \cdots L}=\phi_{(A B \cdots L)} .
$$

The dynamic equations for massless spin $\frac{m}{2}$ field are written as

$$
D^{A A^{\prime}} \phi_{A B \cdots L}=0
$$

The compatibility conditions $[22,23,1]$ of equations (37) for uncharged spinor field (36) can be written as

$$
(m-2) \phi_{A B M(C \ldots K} \Psi_{L)}^{A B M}=0,
$$

where $\Psi_{L A B M}$ is the Weyl spinor on space $V$.

Because on generic curved spaces conditions (38) are not satisfied, there is an essential difficulty in definition of physical fields (36) as solutions of equations (37).

The same difficulty appears and for twistor equations (10), rewritten on space $V$ :

$$
D_{A^{\prime}}^{(A} \omega^{B)}=0,
$$

with compatibility conditions

$$
D^{A^{\prime}(C} D_{A^{\prime}}^{A} \omega^{B)}=-\Psi_{. . D}^{C A . B} \omega^{D}
$$

3.2. Systems of first order partial differential equations. Mentioned field, (37), and twistor, (10), equations are systems of first order partial differential equations. We shall study the general properties of such systems of equations by using methods of the geometrical theory of differential equations [17-20].

Let consider, in general form, a system of first order partial differential equations on space $V^{n}, \operatorname{dim} V^{n}=n$,

$$
f_{s}=\left(x^{1}, \ldots, x^{n}, y^{1}, \ldots, y^{r}, \frac{\partial y^{1}}{\partial x^{1}}, \ldots, \frac{\partial y^{r}}{\partial x^{n}}\right)=0,
$$


where $x^{1}, \ldots, x^{n}$ are independent variables, $y^{1}, \ldots, y^{r}$ are unknown functions and $s=1,2, \ldots, q$. Introducing new unknown variables (functions)

$$
p_{\dot{\alpha}}^{\dot{a}}=\frac{\partial y^{\dot{a}}}{\partial x^{\dot{\alpha}}}, \quad(\dot{a}=1, \ldots, r ; \dot{\alpha}=1, \ldots, n),
$$

we reduce equations (40) to a Pffaf system

$$
\theta^{\dot{a}}=d y^{\dot{a}}-p_{\dot{\alpha}}^{\dot{a}} d x^{\dot{\alpha}}=0,
$$

where variables $p_{\dot{\alpha}}^{\dot{a}}$ satisfy finite relations

$$
f_{s}\left(x^{\dot{\alpha}}, y^{\dot{a}}, p_{\dot{\alpha}}^{\dot{a}}\right)=0 .
$$

Solving (41) on $q$ independent values $p^{\hat{a}}=\left\{p_{\dot{\alpha}}^{\dot{a}}\right\}$ and putting them into (41) we obtain a system of $r$ Pffaf equations on $\hat{r}=r+n r-q$ unknown functions on independent variables $x^{\dot{\alpha}}$ (differentials $d x^{\dot{\alpha}}$ have the role of distinguished variables).

Let $\dot{U}$ be a open region locally isomorphic to $\mathbf{R}^{\hat{r}+n}$. We write the new Pffaf system as

$$
\theta^{\dot{A}}=\theta_{\hat{a}}^{\dot{A}}\left(x^{\hat{\alpha}}, p^{\hat{a}}\right) d p^{\hat{a}}+b_{\dot{a}}^{\dot{A}}\left(x^{\dot{\alpha}}, p^{\hat{b}}\right) d x^{\dot{a}}=0
$$

$\dot{A}=1,2, \ldots, r ; \hat{a}=1,2, \ldots, r+r n-q)$. Equations ( 42) are linearly independent if rang $\left\|\theta_{\hat{a}}^{\dot{A}}\right\| \leq r$ in every point $x^{\dot{\alpha}}$ of open region $U \subset V^{n}$. We mention that integral varieties $I_{\theta}$ of the system (43) should be defined from equations (the closure of (43)):

$$
\begin{gathered}
\theta^{\dot{A}}=0 ; \\
D \theta^{\dot{A}}=0,
\end{gathered}
$$

where quadratic exteriors forms are written as

$$
D \theta^{\dot{A}}=a_{\hat{a} \hat{b}}^{\dot{A}} d p^{\hat{a}} \wedge d p^{\hat{b}}+C_{. \hat{a} \dot{\alpha}}^{\dot{A}} d p^{\hat{a}} \wedge d x^{a}+b_{\dot{a} \dot{b}}^{\dot{A}} d x^{\dot{a}} \wedge d x^{\hat{b}}
$$

If a solution

$$
y^{\dot{a}}=y^{\dot{a}}\left(x^{\dot{\alpha}}\right)
$$

of equations (40) (or equivalently (41) and (42)) is found, it must satisfy integrability conditions

$$
\frac{\partial y^{\dot{a}}}{\partial y^{\dot{\alpha}} \partial x^{\dot{\beta}}}=\frac{\partial y^{\dot{a}}}{\partial x^{\dot{\beta}} \partial y^{\dot{\alpha}}},
$$


or, equivalently,

$$
\partial_{\dot{\beta}} p_{\dot{\alpha}}^{\dot{a}}=\partial_{\dot{\alpha}} p_{\dot{\beta}}^{\dot{a}},
$$

i.e. if equations (40) are compatible, the Pffaf system (41) can be reduced to total differential relations,

$$
d \lambda^{\dot{a}}=d y^{\dot{a}}-p_{\dot{\alpha}}^{\dot{a}} d x^{\dot{\alpha}}=0 .
$$

In this case solution (45) should be obtained from relations

$$
\lambda^{\dot{a}}\left(y^{\dot{b}}, x^{\dot{\alpha}}\right)=C^{\dot{a}}, C^{\dot{a}}=\text { const },
$$

where $\operatorname{rang}\left\|\frac{\partial \lambda^{a}}{\partial y^{b}}\right\| \neq 0$. If conditions (46) are not satisfied, one tries to solve equation (40) by introducing unknown functions

$$
\tilde{p}_{\dot{\alpha}}^{\dot{a}}=p_{\dot{\alpha}}^{\dot{a}}-\frac{\partial \zeta^{\dot{a}}}{\partial x^{\dot{\alpha}}}
$$

and considering a new Pffaf system

$$
\tilde{\theta}^{\dot{a}}=d y^{\dot{a}}-\tilde{p}_{\dot{\alpha}}^{\dot{a}} d x^{\dot{\alpha}}=0,
$$

where $\tilde{f}_{s}\left(x^{\dot{\alpha}}, y^{\dot{a}}\right), \tilde{p}_{\dot{\alpha}}^{\dot{a}}=0$. To obtain a total differential relation we multiply (48) on a nondegenerated matrix function $\mu_{\dot{a}}^{\dot{b}}\left(x^{\dot{\alpha}}, y^{\dot{a}}\right)$ :

$$
d \tilde{\lambda}^{\dot{b}}=\mu_{\dot{a}}^{\dot{b}} d y^{\dot{a}}-\mu_{\dot{a}}^{\dot{b}} \tilde{p}_{\dot{\alpha}}^{\dot{a}} d x^{\dot{\alpha}}=0 .
$$

Integrating system (49) we obtain relations

$$
\tilde{\lambda}^{\dot{b}}\left(x^{\dot{\alpha}}, y^{\dot{a}}\right)=\tilde{C}^{\dot{b}}, \tilde{C}^{\dot{b}}=\text { const },
$$

from which the solution $y^{\dot{a}}=y^{\dot{a}}\left(x^{\dot{\alpha}}\right)$ of equations (40) can be found an explicit form. We note that if deformation functions $\zeta^{\dot{a}}$ from (46) and integrating matrix $\mu_{\dot{a}}^{\dot{b}}$ from (48) exist, the Pffaf system (41) can be expressed as

$$
\theta^{\dot{a}}=d y^{\dot{a}}-p_{\dot{\alpha}}^{\dot{a}} d x^{\dot{\alpha}}=d \zeta^{\dot{a}}+\left(\mu^{-1}\right)_{\dot{b}}^{\dot{a}} d \tilde{\lambda}^{\dot{b}},
$$

where $\left(\mu^{-1}\right)_{\dot{b}}^{a}$ is inverse to $\mu_{\dot{a}}^{\dot{b}}$. As particular cases we can consider the trivial integrating matrix, $\mu_{\dot{a}}^{\dot{b}}=\delta_{\dot{a}}^{\dot{b}}$, and (or) vanishing of deformation when $\zeta^{\dot{a}}=$ const. 
We also emphasize that introducing new, deformed, variables (47) and matrix $\mu_{\dot{a}}^{\dot{b}}$ into relations (42) we obtain new finite relations $\tilde{f}_{s}\left(x^{\dot{\alpha}}, y^{\dot{a}}, p_{\tilde{\alpha}}^{\tilde{a}}\right)=0$ which, as a matter of principle, reflects the deformation of first order partial differential equations (40) into another one ( really for every given system (40) one exists an infinite number of deformations (46) and integrating matrices $\mu_{\dot{a}}^{\dot{b}}$. Considering equations (40) as a fundamental physical equations of type (37), or (39), one arises the problem of definition a unique deformation, motivated from physical and geometrical point of view, transforming mentioned, in general uncompatible on curved spaces, equations into compatible ones, on some auxiliary spaces.

3.3. $N g$-deformations of twistor equations . As was shown twistor equations happen to be uncompatible on a given curved space $V$. Our aim is to formulate an algorithm of transporting mentioned differential equations, by using ng-maps, from the space $V$ to another one, $\underline{V}$, on which compatibility conditions (11) will be satisfied. In this case we extend our system of twistor equations by introducing into consideration additional variables (deformation parameters (15), $a_{\alpha \beta}$ and $b_{\alpha}$, and deformation of connection, $P_{\beta \gamma}^{. \alpha}$, and supplement the initial twistor system on $V$ with a system of basic ng-map equation to $\underline{V}$. Let illustrate this construction for twistor equations (39) rewritten, as a Pffaf system (41),

$$
d \omega^{A}-p^{C^{\prime} C A} d x_{C C^{\prime}}=0,
$$

where unknown functions

$$
p^{C^{\prime} C A}=\frac{\partial \omega^{A}}{\partial x_{C C^{\prime}}}
$$

satisfy finite relations, of type (42),

$$
p^{A^{\prime}(A B)}+\gamma_{. . D}^{A^{\prime}(A \cdot B)} \omega^{D}=0
$$

$\gamma_{. D}^{A^{\prime} A . B}$ are spin-coefficients on space $V$. Twistor equations (39), and their associated Pffaf system (50) and (51), are incompatible on generic curved space-time $V$. We suggest to extend the mentioned system of equations by considering a new system of differential equations

$$
D^{A^{\prime}(A} \tilde{\omega}^{B)}=\Lambda_{. . D}^{A^{\prime}(A . B)} \tilde{\omega}^{D}
$$


or equivalently, a new, associated to (52), Pffaf system

$$
d \tilde{\omega}^{A}-\tilde{p}^{C^{\prime} C A} d x_{C C^{\prime}}=0
$$

where unknown functions $\tilde{p}^{C^{\prime} C A}=\frac{\partial \tilde{\omega}^{A}}{\partial x_{C C^{\prime}}}$ must satisfy relations

$$
\tilde{p}^{A^{\prime}(A B)}+\gamma_{. . D}^{A^{\prime}(A \cdot B)} \tilde{\omega}^{D}+\Lambda_{. . D}^{A^{\prime}(A \cdot B)} \tilde{\omega}^{D}=0 .
$$

Spinors $\Lambda_{. . D}^{A^{\prime} A . B}$ from (52) can be considered as obtained in result of a deformation of type (47) and a multiplication on integrating matrix as in (49). Introducing ng-maps we identify $\Lambda$-spinors with deformation of spin coefficients ${ }^{\star} \gamma_{\mu B}^{. C}$ (see relations $\left.(7)\right)$ :

$$
\Lambda_{A^{\prime} A D}^{B B}=\cdot \gamma_{A A^{\prime} D}^{B}
$$

Proposition 2. Deformed twistor equations (52) ( and associated twistor Pffaf system (53) and (54)) are compatible if spinors (55) solve one of the ng-map equations ((16), (17), (18)-(20) and (21)-(22)) and satisfy one of the corresponding ng-flat criterions ((23), (24), (25) and (28)).

Proof. Defining new spin coefficients

$$
\tilde{\gamma}_{\ddot{A} A^{\prime} D}^{\ldots B}=\gamma_{\ddot{A} A^{\prime} D}^{\ldots B}+\Lambda_{\ddot{A} A^{\prime} D}^{\ldots B}
$$

which according to our proposition become trivial (with vanishing curvature) spin-coefficients on flat space $\tilde{M}$. In this case equations (52) can be written as

$$
\tilde{D}^{A^{\prime}(A} \tilde{\omega}^{B)}=0
$$

Equations (56) are compatible because on the flat space $\tilde{M}$ the Weyl tensor vanishes (see relations (11), (12)). The proposition is proved. $\diamond$

Instead of ng-maps we can consider chains of ng-maps (nc-transforms) $n c: V \rightarrow \underline{M}$. Nc-twistors are defined as solutions of deformed twistor equations with $\Lambda$-spinor, being a superposition of spin tensors,

$$
\Lambda_{A^{\prime} A B}^{\ldots D}={ }^{\star}{ }_{(1)} \gamma_{A A^{\prime} B}^{\ldots D}+{ }^{\star}{ }_{(2)} \gamma_{A A^{\prime} B}^{\cdots D}+\cdots+{ }_{(k)}^{\cdots} \gamma_{A A^{\prime} B}^{\ldots D}
$$

associated to a finite chain of ng-maps. In a particular case when (57) reduces to (55) we obtain ng-twistors. 
3.4. $N g$-images of twistors. On flat space $\underline{M}$ twistors are defined as a pair of spinors, $\underline{Z}^{\alpha}=\left(\underline{\omega}^{A}, \underline{\pi}_{A}\right)$, where

$$
\underline{\omega}^{A}={ }^{(0)} \omega^{A}-i x^{A B^{\prime}(0)} \pi_{B^{\prime}}, \quad \underline{\pi}_{A^{\prime}}={ }^{(0)} \pi_{A^{\prime}}=\text { const },
$$

is the general solution of twistor equations

$$
\underline{D}^{A^{\prime}(A} \underline{\omega}^{B)}=0
$$

Nc-twistors on space $V$, being nc-coimage of space $\underline{M}$, for a given map $n c: V \rightarrow \underline{M}$, are defined as a pair of spinors $Z^{\alpha}=\left(\omega^{A}, \pi_{A^{\prime}}\right)$, where $\omega^{A}$ is the general solution of nc-twistor equations

$$
D^{A^{\prime}(A} \omega^{B)}=\Lambda_{. . D}^{A^{\prime}(A . B)} \omega^{D}
$$

with $\Lambda$-spinors defined from (57). For a local common spinor coordinate system on spaces under consideration we can write $\omega^{A}=\underline{\omega}^{A}$ and define the second spinor $\pi_{A^{\prime}}$ as $\pi_{A^{\prime}}=\frac{i}{2} D_{A A^{\prime}} \omega^{A}$. Taking into account that $\underline{\pi}_{A^{\prime}}=$ $\frac{i}{2} \underline{D}_{A A^{\prime}} \omega^{A}$, we have

$$
\pi_{A^{\prime}}={ }^{(0)} \pi_{A^{\prime}}-\frac{i}{2} \Lambda_{A A^{\prime} C} \omega^{C}
$$

In a similar manner we can define dual nc-twistors on $V$ as pairs of spinors $W_{\alpha}=\left(\lambda_{A}, \mu^{A^{\prime}}\right)$, where

$$
\lambda_{A}={ }^{(0)} \lambda_{A}+\frac{i}{2} \Lambda_{A C^{\prime} A^{\prime}} \mu^{A^{\prime}}, \quad{ }^{(0)} \lambda_{A}=\text { const }
$$

and $\mu^{A^{\prime}}={ }^{(0)} \mu^{A^{\prime}}+i x^{A A^{\prime}(0)} \lambda_{A}$ is the general solution of dual nc-twistor equation

$$
D^{A\left(A^{\prime}\right.} \mu^{\left.B^{\prime}\right)}=\Lambda_{. . D^{\prime}}^{A\left(A^{\prime} \cdot B^{\prime}\right)} \mu^{D^{\prime}}
$$

where spinors $\Lambda_{\ddot{A} A^{\prime} D^{\prime}}$ are defined as a superposition of ng-transformation as (57).

We end this section by concerning the question of geometrical interpretation of nc-twistors . To an isotropic twistor $\underline{Z}^{\alpha}=\left(\underline{\omega}^{A}, \pi_{A^{\prime}}\right) \neq 0, \underline{Z}^{\alpha} \underline{Z}_{\alpha}=$ $0 \quad\left(\underline{\bar{Z}}_{\alpha}=\left(\underline{\bar{\pi}}_{A}, \underline{\bar{\omega}}^{A^{\prime}}\right)\right.$ denotes complex conjugation of $\left.\underline{Z}^{\alpha}\right)$ one associates [2] an isotropic line on space $\underline{M}$ :

$$
x^{a}={ }^{(0)} x^{a}+\eta \underline{\xi}^{a}, \quad \eta_{1}<\eta<\eta_{2},
$$


where ${ }^{(0)} x^{a}=\left(i^{(0)} \underline{\omega}^{B^{\prime}}{ }^{(0)} \underline{\pi}_{B^{\prime}}\right)^{-1}{ }^{(0)} \underline{\omega}^{A}{ }^{(0)} \underline{\omega}^{A^{\prime}}, \underline{\xi}^{a}={ }^{(0)} \underline{\pi}^{A}{ }^{(0)} \underline{\pi}^{A^{\prime}}$. The nccoimages of $\underline{Z}^{\alpha}$ and $\underline{Z}^{\alpha}$ on the space $V$, defined as $Z^{\alpha}=\left(\omega^{A}, \pi_{A^{\prime}}\right)$ and, respectively, as $\bar{Z}_{\alpha}=\left(\bar{\pi}_{A}, \bar{\omega}^{A^{\prime}}\right)$, where

$$
\omega^{A}=\underline{\omega}^{A}={ }^{(0)} \underline{\omega}^{A}-i x^{A A^{\prime}(0)} \underline{\pi}_{A^{\prime}}, \quad \pi_{A^{\prime}}={ }^{(0)} \pi_{A^{\prime}}-\frac{i}{2} \Lambda_{A A^{\prime} C} \omega^{C},
$$

and $\bar{\pi}_{A}={ }^{(0)} \bar{\pi}_{A}-\frac{i}{2} \bar{\Lambda}_{A^{\prime} A C^{\prime}} \bar{\omega}^{C^{\prime}}, \bar{\omega}^{A^{\prime}}=\underline{\bar{\omega}}^{A^{\prime}}$. Using spinors (61) we can verify that

$$
Z^{\alpha} \bar{Z}_{\alpha} \neq 0
$$

i.e. a nc-twistor $Z^{\alpha}$, defined by an isotropic twistor $\underline{Z}^{\alpha}$ is not isotropic. So, nc-twistors on space $V$ parametrize a class of curves on this space, as theirs nc-images (of type (60)) on flat space $\underline{M}$, but, in distinction to usual isotropic twistors, to an nc-twistor one must associate a nearly geodesic on $V$ being a corresponding nc-deformation of a isotropic line in flat space. Really, the nc-image of isotropic line (60) on $\underline{M}$ is a curve $l$ on $V$ ( because of equality (62)) with tangent vector $\xi^{a}=\pi^{A} \bar{\pi}^{A^{\prime}}$ and complementary 2-dimensional distribution defined, for example, by $\xi_{(1)}^{a}=\xi^{b} D_{b} \xi^{a}$ and $\xi_{(2)}^{a}=\xi^{b} D_{b} \xi_{(1)}^{b}$, where $D_{b}=\underline{D}_{b}+{ }^{\star} \underline{D}_{b}, \underline{D}_{b}$ is the deformation of connection associated to map $n c: \underline{M} \rightarrow V$.

\section{Deformation of spinor and twistor struc- tures and generation of curved spaces}

Let on flat space $\underline{M}$ with given primitive spin coefficients $\underline{\gamma}_{A A^{\prime} C}^{\ldots B}$, or connection $\underline{\Gamma}_{\alpha \beta}^{\cdot \cdot \gamma}$, ( with vanishing curvature) is defined a global twistor structure as a solution of twistor equations on $\underline{M}$. Our task is to specify conditions when a deformed twistor structure, obtained as a solution of deformed twistor equations (58) with deformation $\Lambda$-spinor of type (55), will generate a ngflat (pseudo)-Riemannian space-time.

Firstly, we fix a spinor $\Lambda_{A^{\prime} A D}^{B}$. It is still not clear if the new connection $\Gamma_{\ddot{\beta} \gamma}^{\circ}$, defined as to satisfy relations

$$
\Gamma_{\alpha B B^{\prime}}^{\cdots C C C^{\prime}}-P_{\alpha B B^{\prime}}^{\ldots C C C^{\prime}}=\Gamma_{\alpha B B^{\prime}}^{\cdots C C^{\prime}},
$$

where

$$
P_{\alpha B B^{\prime}}^{\ldots C C^{\prime}}=-\Lambda_{\alpha B}^{. C C} \epsilon_{B^{\prime}}^{. C^{\prime}}-\bar{\Lambda}_{\alpha B^{\prime}}^{. C^{\prime}} \epsilon_{B}^{. C}
$$


(for simplicity we consider torsionless connections) will generate both compatible ng-twistor equations (56) and basic ng-map equations associated to a mutual transform $n g: V \rightarrow \underline{M}$. We try to give an answer at this question in the following way. Calculating auxiliary curvature and Ricci tensor for connection (63) and putting both these expressions and deformation tensor (64) into relations (32)-(34) we obtain an algebraic system of equations. If this system is satisfied for some deformation parameters $a_{\alpha \beta}$ and $b_{\gamma}$ (see formulas (15)) it is clear that we have obtained a $\pi_{(1)}$-flat space $V$.

Proposition 3 Deformation spinor $\Lambda_{A A^{\prime} D}^{B}$, and its corresponding deformation tensor $P_{\beta \gamma}^{\ldots \alpha}($ see (64)), will generate a vacuum Einstein field, if and only if, it is compatible this system of partial differential equations:

$$
\partial_{\alpha} P_{\beta \gamma}^{. . \alpha}=2\left(b_{(\alpha} P_{\beta \gamma)}^{. . \alpha}+a_{(\alpha \beta} \delta_{\gamma)}^{\alpha}\right)
$$

$\left(b_{\alpha}\right.$ and $a_{\alpha \beta}$ are some covariant vector and, respectively, symmetric tensor fields).

Proof. We sketch the proof by observing that equations (65) can be obtained by contracting indices $\alpha$ and $\tau$ in equations (18) written for a map $\pi_{(1)}: \underline{M}$, where $R_{\alpha \beta}=0$ and $\underline{R}_{\alpha \beta \gamma \delta}=0$. Of course, to find in explicit form general solutions of equations (65) is also a difficult task. But we can verify, by solving algebraic equations (see considerations from subsection 2.4) if equations (65) are, or not, integrable. $\diamond$

In a similar manner we can analyze the problem of generation of $\pi_{(2)}-$ flat and $\pi_{(3)}$-flat spaces. Let consider, for example, $\pi_{(2)}$-transforms. In this case we shall parametrize the deformation spinor (55) in a form as to induce a deformation tensor of type (18). $\Lambda$-spinors should be also chosen as to induce a deformation tensor (64) satisfying conditions (25) for $\pi_{(2)}{ }^{-}$ maps. Calculating auxiliary curvature and Ricci tensors for connection (63) and putting both mentioned tensors, and taking into account basic $\pi_{(2)}{ }^{-}$ map equations, into expressions (26) and, respectively, (27) we obtain that criterion (25) is an algebraic equation on tensors $R_{\alpha \beta \gamma \delta}, R_{\alpha \beta}, F_{\beta}^{\alpha}$ and covariant vector field $\mu_{\gamma}$.

It is evident that foregoing considerations point to mutual interrelation between integrable deformations of twistor equations and criterions of invariance and integrability of basic equations for ng-maps rather then constitute a method of solution of Einstein equation because explicit constructions of 
metric have not been considered in our study. Perhaps, more convenient for the twistor treatment of gravity is the twistor-gauge formulation of gravity on flat nearly autoparallel backgrounds [7, 12, 14]. The interrelation between nc-twistors and gauge gravity is a matter of our further investigations.

Finally we remark that this paper contains a part of results (on definition of nc-twistors on locally isotropic spaces) communicated at the Coloquium on Differential Geometry (Debrecen, Hungary, 25-30 July, 1994) [24]. There we have also presented some generalizations on spinor and twistor calculus for locally anisotropic spaces (which generalize Lagrange and Finsler spaces) $[25,26]$. The geometric constructions developed in this paper and in $[12,7]$ (in the framework of so-called twistor-gauge treatment of gravity) admit a straightforward extension to locally anisotropic spaces if we apply the formalism of locally anisotropic spinors and twistors $[27,28]$ and use gauge like formulations of locally anisotropic gravity $[26,11,10,13,14]$.

\section{References}

[1] R. Penrose and W. Rindler : Spinors and Space-time, V.1. Cambridge University Press, 1984.

[2] R. Penrose and W. Rindler : Spinors and Spaces-time, V.2. Cambridge University Press, 1984.

[3] G. Vranceanu : Lectii de Geometrie differentiala, V. 2. Ed. Didactica si Pedagogica, Bucuresti, 1977 (in Romanian).

[4] N.S. Sinyukov : Geodesic Maps of Riemannian Spaces, Nauka, Moscow, 1979 (in Russian).

[5] S. Vacaru : Romannian J. of Physics, 39 (1994), 37.

[6] S. Vacaru and S. Ostaf : Buletinul Academiei de Stiinte a Republicii Moldova, fizica si tehnica, 3 (1993), 4.

[7] S. Vacaru : Buletinul Academiei de Stiinte a Republicii Moldova, fizica si tehnica,3 (1993), 17.

[8] S. Vacaru and S. Ostaf : in Lagrange and Finsler Geometry, P. L. Antonelli and R. Miron (eds.), Kluwer Academic Publishers, 1996, 241. 
[9] S. Vacaru and S. Ostaf : Buletinul Academiei de Stiinte a Republicii Moldova, fizica si tehnica, 1 (1994), 64.

[10] S. Vacaru, S. Ostaf and Yu. Goncharenko : Romanian J. Physics, 39 (1994), 199.

[11] S. Vacaru : Contr. Int. Conf. " Lobachevski and Modern Geometry", Part II, V. Bajanov et all (eds.), Kazani, University Press, 1992, 64.

[12] S. Vacaru : Vestnik Mosc. University , 28 (1987), 5 (in Russian).

[13] S. Vacaru : Applications of Nearly Autoparallel Maps and Twistor-Gauge Methods in Gravity and Condensed States, Ph D Thesis. "Al. I. Cuza" University, Iasi, Romania, 1993 (in Romanian).

[14] S. Vacaru : Twistors and Nearly Autoparallel Maps of Curved Spaces (subm. to Rep. Rom. Physics).

[15] K. Yano : Concircular Geometry, I-IV. Proc. Imp. Acad. Tokyo, 16 (1940), 195; 354; 442; 505.

[16] S. A. Schouten and D. Struik : Einfihung in die neucren Medoden den Differentiol Geometrie, Bund 1, 2, 1938.

[17] E. Cartan : Les Systems Differentielles Exterieurs et Lewrs Application Geometricques, Herman and Cie Editeur, Paris, 1945.

[18] M. Haimovichi : Selected Papers, Finsler Geometry, "Al.I. Cuza" Univ., Iasi, Romania, 1984.

[19] S. V. Finnikov : The Method of the Cartan Exterior Differential Forms in Differential Geometry, Gostehizdat, Moscow, 1948 (in Russian).

[20] P.K. Rashevsky : Geometric Theory of Differential Equations, Gostehizdat, Moscow, 1947 (in Russian).

[21] A. Z. Petrov : Einstein Spaces, Pergamon Press, Oxford, 1969.

[22] H. Buchdahl : Nouvo Cim., 10 (1950), 96.

[23] J. Plebanski : Acta Polon., 27 (1965), 361. 
[24] S. Vacaru and S. Ostaf : in Abstracts of Contributions to the Coloquium on Differential Geometry, 25-30 July, 1994, Lajos Kossuth University, Debrecen, Hungary, 1994, 56.

[25] R. Miron and M. Anastasiei : The Geometry of Lagrange Spaces: Theory and Applications, Kluwer Academic Publishers, Dordrecht, Boston, London, 1994.

[26] S. Vacaru and Yu. Goncharenko : Int. J. Theor. Phys., 34, (1995), 1955.

[27] S. Vacaru : Spinor Structures and Nonlinear Connections in Vector Bundles, Generalized Lagrange and Finsler Spaces (to appear in J. Math. Phys., 36, N 10 or 12, 1995).

[28] S. Vacaru : Spinors in Multidimensional and Locally Anisotropic Spaces (in preparation). 